\title{
Pen Is Mightier than the Sword: Columbia Shows the Way in Formulating Neurotrauma Guidelines
}

\author{
Deepak Agrawal ${ }^{1}$ \\ ${ }^{1}$ Department of Neurosurgery and Gamma Knife, All India institute
of Medical Sciences, New Delhi, India
}

J Neurosci Rural Pract 2020;11:1

The saying "one shoe does not fit all" is especially true for traumatic brain injuries (TBI) which occur most frequently in resource constrained low- and middle-income countries (LMICs), yet manage patients based on guidelines emanating from high-income countries (HICs). A prime example is intracranial pressure (ICP) monitoring. In countries like India where majority of the population is uninsured or poorly insured, more than $90 \%$ of all severe TBl's are managed without ICP monitoring, simply because the cost of ICP catheter is prohibitive to most neurosurgical centres. ${ }^{1,2}$ Despite practical issues like this, the national societies of these countries continue to rubber stamp the brain trauma foundation guidelines, ${ }^{3}$ without looking at the ground realities in the country.

It is therefore a welcome change to see a position paper coming out from Columbia which shows a new approach to manage TBIs based on the resources available. ${ }^{4}$ It is important that guidelines for TBIs reflect the local resources and therefore this "two-dimensional" concept of pairing protocols with the resource setting is commendable and needs to be emulated by other developing countries like India.

I believe that these guidelines should be adopted by bodies such as World Health Organization (WHO) and World Federation of Neurosurgical Societies (WFNS), as a template for developing countries and fine-tuned for each specific country. For example, in India, jugular bulb venous oxygen saturation $\left(\mathrm{SjvO}_{2}\right)$ and electroencephalography (EEG) monitoring are not done, even in tertiary setting, and may be removed from India-specific guidelines.

In absence of proactive role of government in countries like India, the onus falls on national societies, like Neurotrauma
Society of India, to frame guidelines after a similar consensus BOOTStraP exercise, ${ }^{4}$ have it ratified by regulatory authorities like National Medical Commission in India, then have them enforced across the country. This will go a long way in having consistency of neurotrauma care based on resource setting and pave the way for other specialties to similarly follow.

I again commend the stakeholders in Columbia for coming together to crystallize their discussions into this well written position paper which, in fact, is a legacy for the people of Columbia (and a template for other countries), and I am sure its citizens will benefit from this in the very near future.

\section{Conflict of Interest}

None declared.

\section{References}

1 Chesnut RM, Temkin N, Carney N, et al; Global Neurotrauma Research Group. A trial of intracranial-pressure monitoring in traumatic brain injury. N Engl J Med 2012;367(26):2471-2481

2 Joseph M. Intracranial pressure monitoring: vital information ignored. Indian J Crit Care Med 2005;9:35-41

3 Carney N, Totten AM, O'Reilly C, et al. Guidelines for the management of severe traumatic brain injury, fourth edition. Neurosurgery. 2017;80(1):6-15

4 Rubiano AM, Vera DS, Montenegro JH, et al. Recommendations of the Colombian consensus committee for the management of traumatic brain injury in prehospital, emergency department, surgery, and intensive care (beyond one option for treatment of traumatic brain injury, a stratified protocol (BOOTStraP). J Neurosci Rural Pract 2020;11(1):7-22

\author{
Address for correspondence \\ Deepak Agrawal, MBBS, MS, MCh, \\ Department of Neurosurgery and \\ Gamma Knife, All India institute of \\ Medical Sciences, New Delhi, India \\ (e-mail: drdeepak@aiims.edu).
}

DOI https://doi.org/

10.1055/s-0040-1701777 ISSN 0976-3147.
(C)2020 Association for Helping

Neurosurgical Sick People
License terms

$(\circledast) \Theta \circledast$ 